MALARIA

JOURNAL

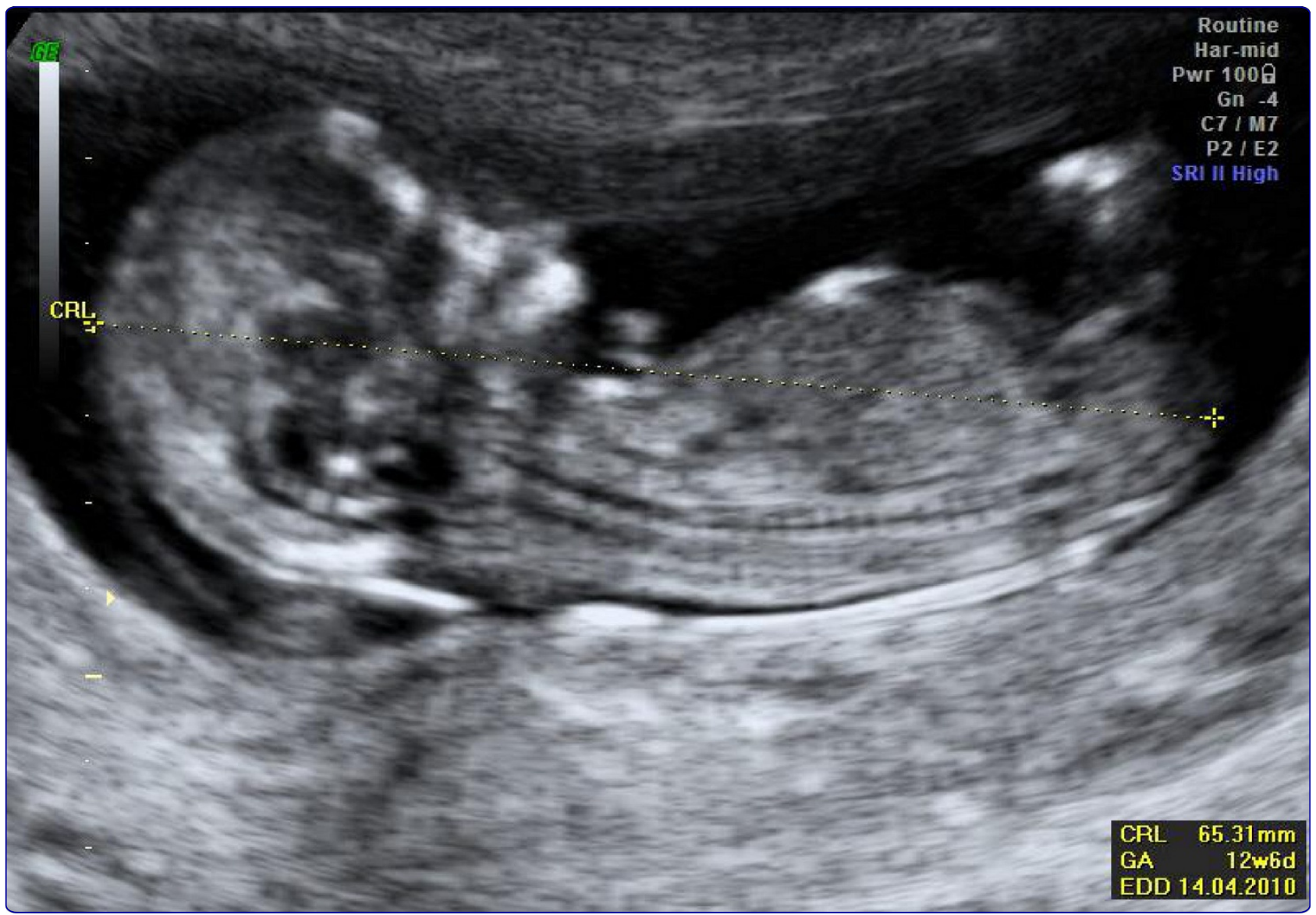

Effect of malaria in pregnancy on foetal cortical brain development: a longitudinal observational study

Rijken et al.

C Biomed Central 


\title{
Effect of malaria in pregnancy on foetal cortical brain development: a longitudinal observational study
}

\author{
Marcus J Rijken ${ }^{1 * \dagger}$, Merel Charlotte de Wit ${ }^{2+}$, Eduard JH Mulder ${ }^{2}$, Suporn Kiricharoen ${ }^{1}$, Noaeni Karunkonkowit ${ }^{1}$, \\ Tamalar Paw ${ }^{1}$, Gerard HA Visser ${ }^{2}$, Rose McGready ${ }^{1,3,4}$, François H Nosten ${ }^{1,3,4}$ and Lourens R Pistorius ${ }^{2}$
}

\begin{abstract}
Background: Malaria in pregnancy has a negative impact on foetal growth, but it is not known whether this also affects the foetal nervous system. The aim of this study was to examine the effects of malaria on foetal cortex development by three-dimensional ultrasound.

Methods: Brain images were acquired using a portable ultrasound machine and a 3D ultrasound transducer. All recordings were analysed, blinded to clinical data, using the $4 \mathrm{D}$ view software package. The foetal supra-tentorial brain volume was determined and cortical development was qualitatively followed by scoring the appearance and development of six sulci. Multilevel analysis was used to study brain volume and cortical development in individual foetuses.

Results: Cortical grading was possible in 161 out of 223 (72\%) serial foetal brain images in pregnant women living in a malaria endemic area. There was no difference between foetal cortical development or brain volumes at any time in pregnancy between women with immediately treated malaria infections and non-infected pregnancies.

Conclusion: The percentage of images that could be graded was similar to other neuro-sonographic studies. Maternal malaria does not have a gross effect on foetal brain development, at least in this population, which had access to early detection and effective treatment of malaria.
\end{abstract}

Keywords: Malaria, Pregnancy, Ultrasonography, Prenatal, Brain, Foetus, Cerebral cortex

\section{Background}

Both Plasmodium vivax and Plasmodium falciparum malaria are associated with maternal and foetal morbidity and mortality $[1,2]$. Malaria in pregnancy causes a decrease in birth weight by intra-uterine growth restriction (IUGR), preterm delivery, or both [3]. Malaria infection in the first half of pregnancy is associated with a reduction in foetal head diameter [4] and maternal P. falciparum malaria changes utero-placental haemodynamics [5]. Whether in-utero exposure to malaria has an effect on the growth and development of the foetal central nervous system is not known.

Studying the pathophysiological consequences of malaria in pregnancy on the foetus has been complicated by

\footnotetext{
*Correspondence: marcus@shoklo-unit.com

${ }^{\dagger}$ Equal contributors

'Shoklo Malaria Research Unit, PO Box 46, Mae Sot, Tak 63110, Thailand

Full list of author information is available at the end of the article
}

unreliable diagnosis of (early) pregnancy infections, difficulties in gestational age (GA) estimation and use of various methods to measure newborn anthropometrics [6]. The primary cortical folding process in the newborn is an early marker for functional neuro-development [7]. Growth restricted foetuses show a faster cortical folding process in comparison to normal foetuses, when measured with magnetic resonance imaging [7] or threedimensional (3D) ultrasound (C. Businelli, unpublished data). Such increased cortical maturation might be related to the functional disturbances (e.g. lower IQ, attention deficit hyperactivity disorder) found in children affected by IUGR $[8,9]$. The relatively new technique of 3D-ultrasound imaging of the foetal brain has rarely before been available in malaria endemic areas. The aim of this pilot study was to examine the effects of malaria in pregnancy on foetal brain development. The hypothesis 
was that foetuses affected by maternal malaria experience growth restriction, including smaller foetal brain volume and an accelerated cortical folding process.

\section{Methods}

Population

The participants in this study were attending the antenatal clinic (ANC) at Shoklo Malaria Research Unit (SMRU), which is located on the Thai-Burmese border where malaria transmission is low and seasonal [10]. Since 1986, the SMRU runs an ANC programme including weekly malaria screening to detect and treat all parasitaemic episodes during pregnancy to prevent maternal deaths [10]. There is no presumptive treatment of malaria or chemoprophylaxis. Every woman with a malaria episode detected by peripheral smear is immediately treated using WHO protocols: chloroquine for P.vivax infections in any trimester and artemisinin-based combination therapy for P.falciparum, and quinineclindamycin for first trimester infections [11]. Routine antenatal ultrasound performed by locally trained health workers commenced in 2001 [12]. All women are encouraged to attend the ANC as early as possible in pregnancy and to deliver at SMRU under the care of Advance Life Support in Obstetrics (ALSO) trained midwives and doctors; those requiring caesarean section are transferred to the nearest Thai hospital. In this study, pregnant women with documented $P$. falciparum or $P$. vivax parasitaemia were included. They were compared with uninfected women matched for foetal gender, parity, and maternal age (within five years).

\section{Ultrasound scans}

Ultrasound scans were performed trans-abdominally using a General Electric Voluson i (GE Healthcare, Austria) with a RAB2-5-RS, $2-5 \mathrm{MHz}$ real-time $4 \mathrm{D}$ probe. The machine was housed in a dedicated air-conditioned room. All scans were obtained by locally trained sonographers specifically skilled in advanced foetal growth scanning (SK and NK) or a resident in obstetrics certified in antenatal ultrasound scanning (MJR), with regular internal quality control at SMRU. In addition, all images were sent for external quality control to the INTERGROWTH-21st Project team at the University of Oxford [13]. Foetal crown rump length (CRL) between $9^{+0}$ and $13^{+6}$ weeks was used to define gestational age (GA). Thereafter, women were invited to attend a foetal growth scan every five weeks until delivery to take $2 \mathrm{D}$ foetal biometric measurements and 3D sweeps, including the foetal head at the mid-ventricular plane. The volume box size and sweep angle were adapted to include the entire head. Care was taken to minimize movement artifacts. Once the scan was complete, volume data were stored for later analysis.
Table 1 Maternal and newborn characteristics

\begin{tabular}{llll}
\hline & $\begin{array}{l}\text { No malaria } \\
(\boldsymbol{n}=\mathbf{2 2})\end{array}$ & $\begin{array}{l}\text { Malaria } \\
(\boldsymbol{n}=\mathbf{2 2})\end{array}$ & $\mathbf{P}$ \\
\hline Pregnant women & & & \\
\hline Age (yrs) & $27.0(19-39)$ & $25.5(19-38)$ & 0.23 \\
\hline Nulliparous & $3(14)$ & $3(14)$ & 1.0 \\
\hline Gravida & $3(1-10)$ & $3(1-7)$ & 0.36 \\
\hline Parity & $2(0-5)$ & $2(0-5)$ & 0.82 \\
\hline Height (m) & $1.53(1.46-1.64)$ & $1.53(1.44-1.61)$ & 0.71 \\
\hline Weight (kg) & $50.5(39-70)$ & $46.5(39-61)$ & 0.20 \\
\hline Weight gain (kg) & $7.5(-2-17)$ & $9(4-15)$ & 0.76 \\
\hline BMI (kg m-2) & $21.5(17.8-30.2)$ & $20.1(17.1-27.6)$ & 0.15 \\
\hline MUAC (cm) & $25.6(14.5-32.0)$ & $25.0(20.6-30.6)$ & 0.81 \\
\hline Smoking & $3(14)$ & $7(32)$ & 0.28 \\
\hline NOC & $25.5(12-34)$ & $26.5(15-33)$ & 0.48 \\
\hline Father's age & $29.5(19-43)$ & $29.5(24-38)$ & 0.80 \\
\hline Other infections & $3(14)$ & $5(23)$ & 0.70 \\
\hline Anaemia & $7(32)$ & $14(64)$ & 0.07 \\
\hline Severe anaemia & $0(0)$ & $3(14)$ & 0.23 \\
\hline Early pre-eclampsia & $2(9)$ & $2(9)$ & 1.0 \\
\hline Newborns & & $279(261-293)$ & 0.58 \\
\hline Gestational Age (days) & $277(241-295)$ & $36.4 \%$ & 1.0 \\
\hline Sex (\% boys) & $36.4 \%$ & $2685(1940-3410)$ & 0.18 \\
\hline Weight (grams)* & $2840(1720-3660)$ & 0.88 \\
\hline Length (cm)* & $49.0(44.6-51.0)$ & $49.4(45.0-51.3)$ & 0.53 \\
\hline Head circumference & $32.0(29.5-39.3)$ & $32.0(31.0-35.1)$ & \\
(cm)* & & & \\
\hline De & & & \\
\hline
\end{tabular}

Data are presented as median (range) or as number (\%). MUAC mid upper arm circumference, NOC number of consultations. * Data shown of newborns weighed within 24 hours of delivery; 17 and 18 in the no malaria and malaria group, respectively.

\section{Definitions}

Malaria was diagnosed by Giemsa stained thick and thin blood films; 200 fields on the thick film were read before being declared negative. Severe malaria was defined according to WHO treatment guidelines [11]. Birth weight analysis was confined to life born, congenitally normal, singleton infants weighed in the first 24 hrs of life. Prematurity was defined as delivery before $37^{+0}$ weeks' gestation. Birth weight, length, and head circumference were measured twice by the trained and quality controlled anthropometry team on electronic Seca baby scales (Model 376, accuracy 10 grams), a Harpenden Infantometer with digital counter readings to one $\mathrm{mm}$, or Seca Head Circumference Tape accurate to one $\mathrm{mm}$, respectively, the first two being calibrated twice a week [6]. All ultrasound and anthropometric methods were identical to the INTERGROWTH- $21^{\text {st }}$ study protocol [13].

One author $(\mathrm{MCW})$, blinded to any clinical data, analysed all recorded volumes using the $4 \mathrm{D}$ view software package, version 9.1 (GE healthcare). The foetal supra- 


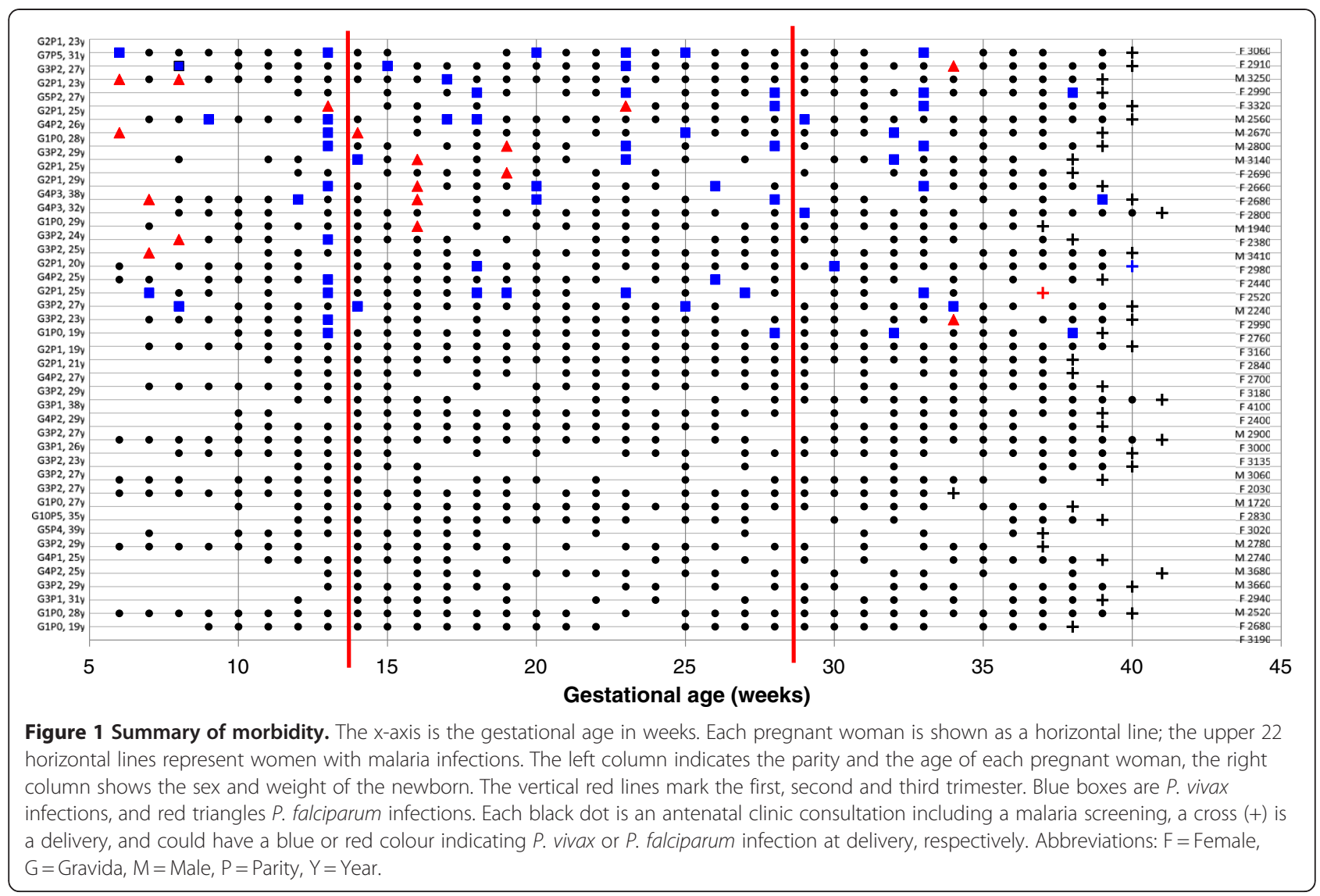

tentorial brain volume was determined and cortical development was qualitatively followed by scoring the appearance and development of six sulci (the Sylvian fissure and the superior temporal, central, parietooccipital, calcarine and cingulate sulcus) [14]. A grading system was used to systematically assess the development of every sulcus independently. The depth and ramification of the specific sulcus was scored in a range from zero to five, where zero equals "not visible" and five "fully developed", as described previously [14]. Sulci on both the left and right side of the brain were graded, and the mean grade was used for analysis.

\section{Statistical analysis}

Clinical data and the results of the ultrasound scans were entered into a Microsoft Access database and

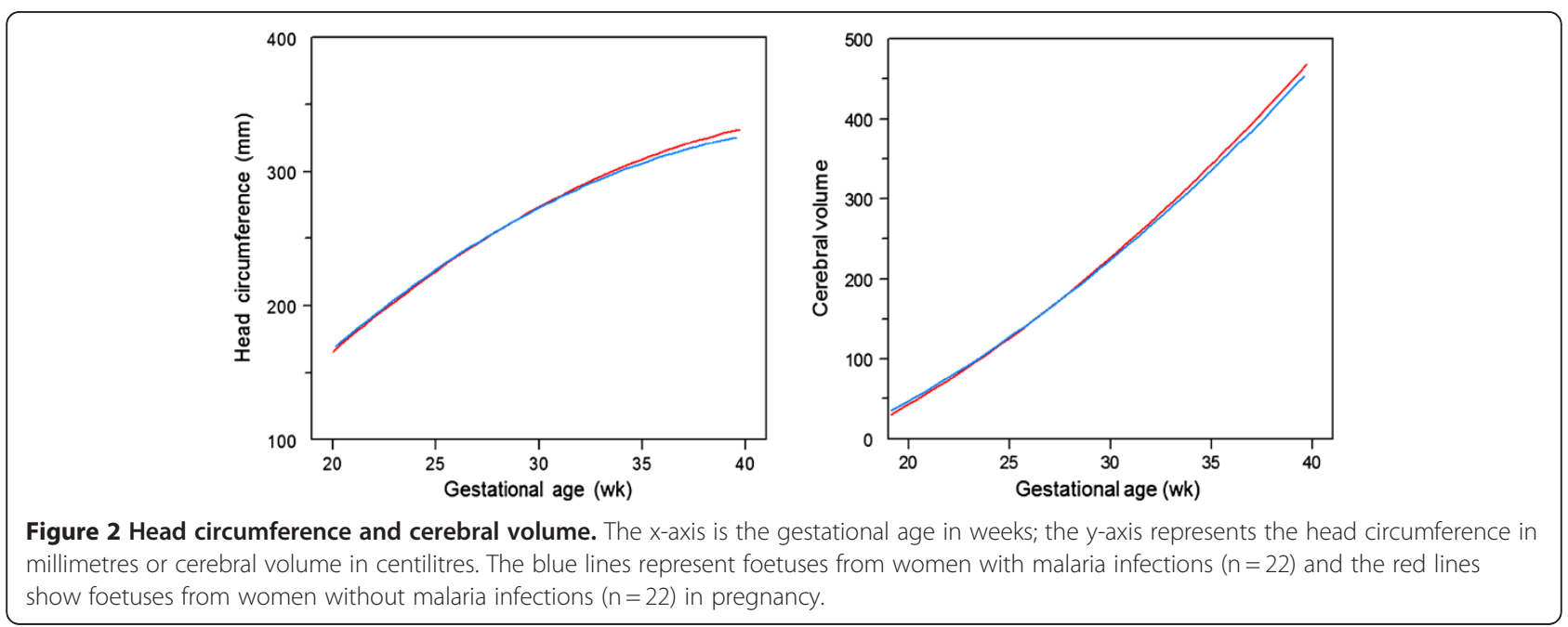


Table 2 Time (in days) of first appearance, interval and full development of cortical sulci

\begin{tabular}{lllll}
\hline Sulcus & Group & First appearance & $\mathbf{p}$ & Maximal grade \\
\hline Sylvian & Malaria & $115(99-131)$ & 0.539 & $230(204-268)$ \\
\hline & Control & $117(101-134)$ & & $231(204-272)$ \\
\hline Superior Temporal & Malaria & $145(118-179)$ & 0.362 & $243(211-268)$ \\
\hline & Control & $150(112-177)$ & $238(172-263)$ \\
\hline Parieto-occipital & Malaria & $123(99-147)$ & 0.422 & $240(204-277)$ \\
\hline Central & Control & $120(98-146)$ & $244(216-274)$ \\
\hline & Malaria & $145(104-167)$ & $221(201-268)$ \\
\hline Calcarine & Control & $150(112-169)$ & 0.429 & $219(191-241)$ \\
\hline & Malaria & $126(99-151)$ & $226(204-226)$ \\
\hline Cingulate & Control & $133(109-172)$ & 0.282 & $224(202-263)$ \\
\hline & Malaria & $145(106-179)$ & $248(217-277)$ \\
\hline
\end{tabular}

Data are presented as median (range).

analysed using SPSS version 17 for Windows. The Mann-Whitney, Chi-square or Fisher's exact test were used for comparison of ranks or categorical data, as appropriate. Multilevel analysis was used to study brain volume and cortical development in individual foetuses, with grades considered as a continuous variable [14]. The significance level was set at $\alpha=0.05$.

\section{Ethical approval}

This study was part of a larger foetal growth project (ClinicalTrials.gov Identifier: NCT00840502), approved and yearly renewed by the Ethics Committees of Oxford (OxTREC (14-08)) and Mahidol (TMEC 2008-028) universities. All women provided written informed consent.

\section{Results}

In total 215 women were recruited between February 2009 and August 2010. Of these 22 women were diagnosed with malaria infections: 14 were infected with $P$. falciparum malaria and eight women with $P$. vivax. The characteristics of both infected and non-infected women are shown in Table 1. The frequency and timing of the infections are shown in Figure 1. Most malaria episodes were uncomplicated (77/78; 98.7\%). One pregnant woman had severe malaria during labour. There were more anaemic women in the malaria group, as expected. Three of these women required a blood transfusion. Other morbidities in pregnancy are summarized in Table 1.

\section{Cortical maturation and supra-tentorial brain volume}

In total, 223 brain images were analysed: 113 in the malaria group and 110 in the non-infected group. The median number of brain images per foetus was five (range 4-6) and did not differ between both groups. In the malaria affected group, $73.4 \%(83 / 113)$ of images could be visualized well enough to grade them reliably. In the non-infected group this percentage was similar (70.9\%; $78 / 110), p=0.78$. The presence of reverberation artefacts was the most important reason for the inability to visualize a sulcus in the hemisphere closest to the abdominal wall. Furthermore, in early pregnancies, foetal motion artefacts often troubled visualization. The head circumference and brain volumes between malaria infected and uninfected pregnancies, which were not significantly different, are illustrated in Figure 2. Table 2 shows the mean GA at first appearance and full foetal development per sulcus. All six sulci developed similarly between the two groups (Figure 3, Table 2). The median number of days between the first appearance of any sulcus and the first fully matured sulcus in the same foetus was 105 for both malaria [range 79-133] and non-malaria [range $70-139$ ] groups $(p=0.21)$. Only the cingulate sulcus initially matured significantly faster in foetuses affected by maternal malaria in pregnancy in comparison to foetuses of malaria-free pregnancies (Figure 3). There was no significant association between the number of episodes, species, timing of infection, or number of previous pregnancies and cortical maturation or volumes.

\section{Newborns}

Eleven (25\%) women delivered at home, one (2\%) underwent a caesarean section because of prolonged labour and the remaining 32 (73\%) delivered in the SMRU clinic. There were no stillbirths, and all newborns appeared congenitally normal, although one infant from the non- malaria group was diagnosed with congenital heart disease later in life. Overall, the median birth weight was 2,780 [range 1,720 - 3,660] grams in newborns weighted within 24 hours after delivery $(n=35)$, and the gestational age at delivery was $39^{+3}$ [range $33^{+3}$ 

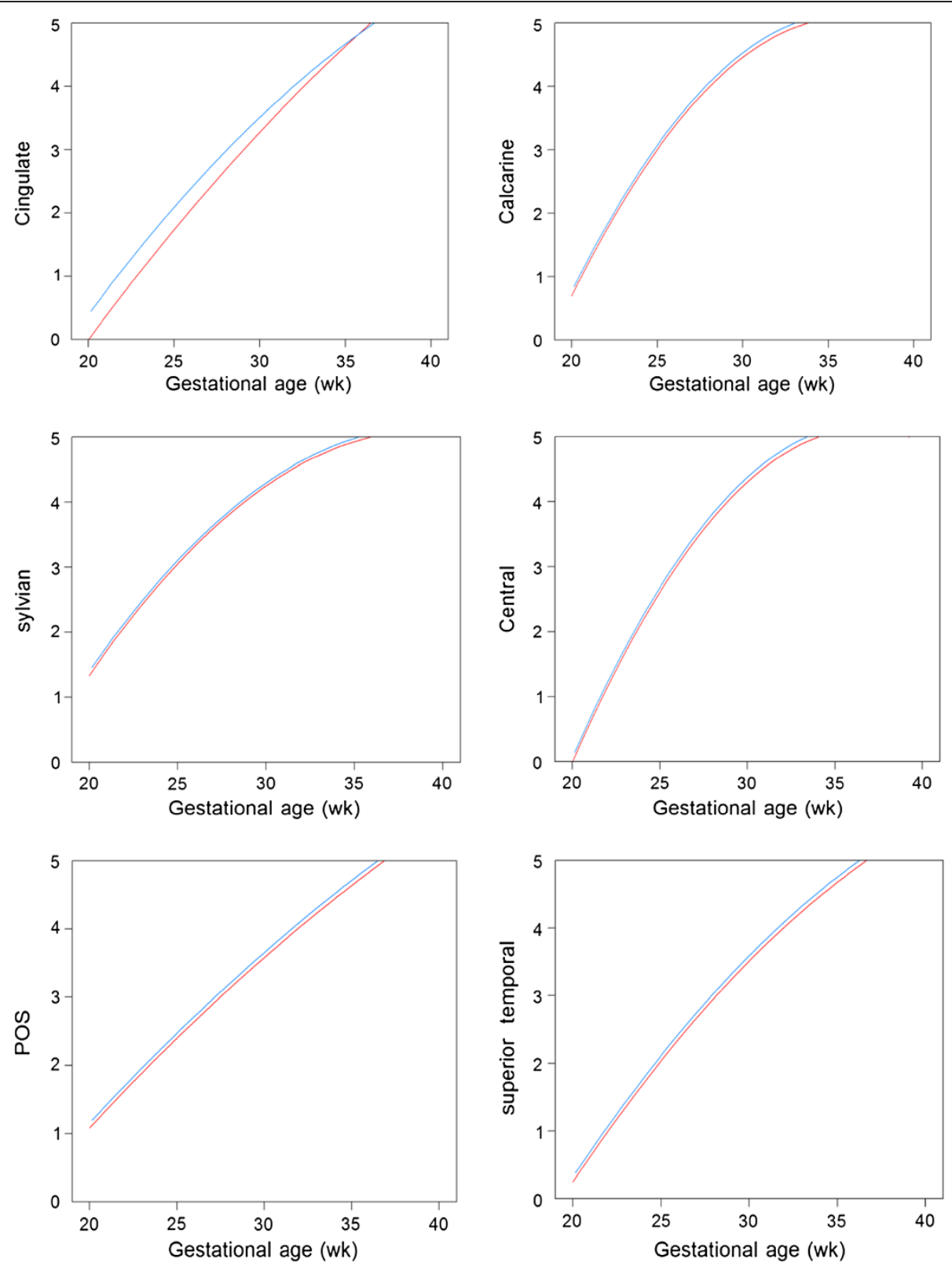

Figure 3 Development of foetal cortex (Sylvian fissure, superior temporal, central, parieto-occipital, calcarine and cingulate sulcus). The $x$-axis is the gestational age in weeks; the $y$-axis represents the grading of the Sylvian fissure, superior temporal, central, parieto-occipital, calcarine and cingulate sulcus. The blue lines represent foetuses from women with malaria infections $(n=22)$ and the red lines show foetuses from women without malaria infections $(n=22)$ in pregnancy.

$-41^{+2}$ ] weeks, including one premature neonate. All anthropometric measurements in the newborn were similar between the two groups (Table 1).

\section{Discussion}

In this study, the foetal brain volumes and foetal cortex development were compared between women with and without malaria. Although the images of this study were not primarily obtained for neuro-sonographic evaluations and 3D ultrasound exposure time was limited, seventy percent of sulci could be visualized well enough to be graded. This percentage is similar to a previous study in healthy volunteers, where ultrasound scans were performed by a neuro-sonography expert [14]. In the busy malaria clinics, there was no possibility for realtime evaluation of the quality of obtained images. This is the first study to address the effect of maternal malaria on foetal cortical development or supra-tentorial volume. No difference in brain volume expansion or foetal cortical folding in general at any time in pregnancy 
between women with immediately treated malaria infections and non-infected pregnancies was detected. Of all graded sulci, only the cingulate sulcus matured faster in foetuses of women affected by malaria during pregnancy. The significance of a difference in maturation of a single sulcus has to be interpreted cautiously in the analysis of the general cortical development. Although the sample size of this pilot study is small, the blinding of the sonographers and observers to the malaria status of the mother, and the well matched groups may allow preliminary conclusions that maternal malaria does not have a gross effect on foetal brain development, at least in this population which had access to early detection and effective treatment of malaria.

The timing of malaria infection has an impact on the growth and development of the foetus [15]. The small sample size did not allow sub-analysis of groups infected in certain trimesters in pregnancy, nor of effect of symptoms or malaria species separately, but most women $(73 \%, 16 / 22)$ were infected as early as the first trimester and most women had malaria infections throughout pregnancy. Similar studies with larger sample sizes in different malaria endemic settings may be needed to confirm these findings. Although three dimensional ultrasound machines are delicate and expensive and not available in most malaria endemic settings, this tool may be helpful in determining the impact of malaria on the foetal nervous system and indicating the newborns neurodevelopment.

In conclusion, maternal malaria does not have a gross effect on foetal brain development, at least in this population, which had access to early detection and effective treatment of malaria.

\section{Competing interest}

The authors declare that they have no competing interests.

\section{Authors' contributions}

MJR designed the study, carried out the ultrasound scanning, performed the statistical analysis and drafted the manuscript. MCW performed the brain volume measurements and cortex grading, performed the statistical analysis and drafted the manuscript. EJHM carried out the statistical analysis and helped to draft the manuscript. SK, NK and TP performed the ultrasound scanning, organized and coordinated the study on site. GHV participated in the design of the study and revision of the manuscript. RMG participated in the design and coordination of the study, helped in the statistical analysis and revised the manuscript. FN conceived of the study and participated in the design and coordination of the study, and revised the manuscript. LRP participated in the design and analysis of the study, organized the image analysis and helped to draft and revised the manuscript. All authors read and approved the final manuscript.

\section{Acknowledgements}

We would like to thank the patients and staff of SMRU for their contribution, especially William Moroski, Wah Say, Esther, Suthatsana Rungwilailaekhiri (Miss Snow), December Win and Khin Nwet Soe. The SMRU is supported by The Wellcome Trust of Great Britain, as part of the Oxford Tropical Medicine Research Programme of Wellcome Trust-Mahidol University. The Christophe and Rodolphe Mérieux Foundation supported the study through a prize (2008) to FN. The funding sources were not involved in the collection, analysis and interpretation of the data, the writing of the article or in submission of the paper for publication.

\section{Author details}

${ }^{1}$ Shoklo Malaria Research Unit, PO Box 46, Mae Sot, Tak 63110, Thailand. ${ }^{2}$ Department of Obstetrics, University Medical Center Utrecht, Utrecht, Netherlands. ${ }^{3}$ Centre for Tropical Medicine, Churchill Hospital, Oxford, UK. ${ }^{4}$ Faculty of Tropical Medicine, Mahidol University, Bangkok, Thailand.

\section{Received: 27 February 2012 Accepted: 25 May 2012}

Published: 2 July 2012

\section{References}

1. Rijken MJ, McGready R, Boel ME, Poespoprodjo R, Singh N, Syafruddin D, Rogerson S, Nosten F: Malaria in pregnancy in the Asia-Pacific region. Lancet Infect Dis 2012, 12:75-88.

2. Desai M, ter Kuile FO, Nosten F, McGready R, Asamoa K, Brabin B, Newman RD: Epidemiology and burden of malaria in pregnancy. Lancet Infect Dis 2007, 7:93-104.

3. Umbers AJ, Aitken EH, Rogerson SJ: Malaria in pregnancy: small babies, big problem. Trends Parasitol 2011, 27:168-175.

4. Rijken MJ, Papageorghiou AT, Thiptharakun S, Kiricharoen S, Dwell SL, Wiladphaingern J, Pimanpanarak M, Kennedy SH, Nosten F, McGready R: Ultrasound evidence of early fetal growth restriction after maternal malaria infection. PLoS One 2012, 7:e31411.

5. Dorman EK, Shulman CE, Kingdom J, Bulmer JN, Mwendwa J, Peshu N, Marsh K: Impaired uteroplacental blood flow in pregnancies complicated by falciparum malaria. Ultrasound Obstet Gynecol 2002, 19:165-170.

6. Rijken M, Rijken J, Papageorghiou A, Kennedy S, Visser G, Nosten F, McGready R: Malaria in pregnancy: the difficulties in measuring birthweight. BJOG 2011, 118:671-678.

7. Dubois J, Benders M, Borradori-Tolsa C, Cachia A, Lazeyras F, Ha-Vinh Leuchter R, Sizonenko SV, Warfield SK, Mangin JF, Huppi PS: Primary cortical folding in the human newborn: an early marker of later functional development. Brain 2008, 131:2028-2041.

8. Strang-Karlsson S, Raikkonen K, Pesonen AK, Kajantie E, Paavonen EJ, Lahti J, Hovi P, Heinonen K, Jarvenpaa AL, Eriksson JG, Andersson S: Very low birth weight and behavioral symptoms of attention deficit hyperactivity disorder in young adulthood: the Helsinki study of very-low-birth-weight adults. Am J Psychiatry 2008, 165:1345-1353.

9. Geva R, Eshel R, Leitner Y, Valevski AF, Harel S: Neuropsychological outcome of children with intrauterine growth restriction: a 9-year prospective study. Pediatrics 2006, 118:91-100.

10. Nosten F, ter Kuile F, Maelankirri L, Decludt B, White NJ: Malaria during pregnancy in an area of unstable endemicity. Trans $R$ Soc Trop Med Hyg 1991, 85:424-429.

11. WHO: Guidelines for the treatment of malaria. 2nd edition. Geneva: World Health Organization; 2010.

12. Rijken MJ, Lee SJ, Boel ME, Papageorghiou AT, Visser GH, Dwell SL, Kennedy SH, Singhasivanon P, White NJ, Nosten F, McGready R: Obstetric ultrasound scanning by local health workers in a refugee camp on the Thai-Burmese border. Ultrasound Obstet Gynecol 2009, 34:395-403.

13. International Fetal and Newborn Growth Consortium (INTERGROWTH-21st). [https://www.medscinet.net/intergrowth/protocol.aspx] [2 September 2011].

14. Pistorius LR, Stoutenbeek P, Groenendaal F, de Vries L, Manten G, Mulder E, Visser G: Grade and symmetry of normal fetal cortical development: a longitudinal two- and three-dimensional ultrasound study. Ultrasound Obstet Gynecol 2010, 36:700-708.

15. Huynh BT, Fievet N, Gbaguidi G, Dechavanne S, Borgella S, Guezo-Mevo B, Massougbodji A, Ndam NT, Deloron P, Cot M: Influence of the timing of malaria infection during pregnancy on birth weight and on maternal anemia in Benin. Am J Trop Med Hyg 2011, 85:214-220.

doi:10.1186/1475-2875-11-222

Cite this article as: Rijken et al:: Effect of malaria in pregnancy on foetal cortical brain development: a longitudinal observational study. Malaria Journal 2012 11:222. 